\title{
Implantação de Acessibilidade em Organizações de Software
}

\author{
Débora M. Barroso Paiva ${ }^{1}$, Lucinéia Souza Maia ${ }^{1}$, Renata P. de Mattos Fortes ${ }^{2}$, \\ Marcelo A. Santos Turine ${ }^{1}$, André Pimenta Freire ${ }^{3}$ \\ ${ }^{1}$ Departamento de Computação e Estatística \\ Universidade Federal do Mato Grosso do Sul \\ Caixa Postal 549 - 79.070-900 - Campo Grande - MS - Brasil \\ ${ }^{2}$ Departamento de Ciências de Computação \\ Instituto de Ciências Matemáticas e de Computação - Universidade de São Paulo \\ Caixa Postal 668 - 13.560-970 - São Carlos - SP- Brasil \\ ${ }^{3}$ Department of Computer Science \\ University of York \\ York, UK - YO105DD \\ \{debora, lucineiamaia, turine\}@dct.ufms.br, renata@icmc.usp.br, apfreire@cs.york.ac.uk
}

\begin{abstract}
The technological revolution has enabled the diffusion of information in a very fast fashion. Besides information technology has become an important platform for business, education, and entertainment. However, problems related to the accessibility of content have been a preponderant factor to prevent users with disabilities to be included in such environments. In this context, this mini-course intends to explore elements of accessibility of software systems and, mainly, the activities that an organization needs to accomplish to implement accessibility for their information systems.
\end{abstract}

Resumo. A revolução tecnológica tem promovido a disseminação da informação cada vez mais precisa e rápida, além de se tornar um importante meio de negócio, educação e entretenimento. Porém, problemas relacionados à acessibilidade do conteúdo tem sido um fator preponderante para a exclusão de pessoas com deficiência. Neste contexto, este mini-curso pretende explorar elementos de acessibilidade de sistemas de software e, principalmente, as atividades que uma organização deve cumprir para a institucionalização da acessibilidade.

\section{Acessibilidade Web}

A Internet oferece às pessoas acesso a informações e serviços. Porém, esta tecnologia não pode atingir todo seu potencial se projetistas e desenvolvedores não seguirem as regras e recomendações de acessibilidade. De fato, a acessibilidade é uma importante vantagem para todos os usuários, não somente para pessoas com dificuldades de acesso ou portadores de deficiência [PÜHRETMAIR e MIESENBERGER, 2005].

O princípio chave da acessibilidade Web é projetar aplicações Web que sejam flexíveis para reunir diferentes necessidades, preferências e situações do usuário. Ao considerar a acessibilidade, é possível (1) fazer com que pessoas com deficiência e idosos tornem-se independentes ao usarem a Internet; (2) que a aplicação esteja mais 
adequada a leis e regulamentações de acessibilidade e (3) a audiência do site seja aumentada [W3C/WAI, 1997; PADDISON e ENGLEFIELD, 2003].

Os usuários com deficiência física podem ter dificuldade em usar dispositivos apontadores, usar o teclado (exemplo: pressionar duas teclas ao mesmo tempo) ou programas que requerem uma resposta em período de tempo específico. Pessoas com deficiência visual têm problemas com informação mostrada na tela, com o uso de dispositivos apontadores que requerem coordenação dos olhos-mãos (como o mouse). As pessoas com limitações na audição têm dificuldade para detectar sons ou distinguir informações com barulho de plano de fundo, outro problema é encontrado quando a aplicação exige entrada de voz, porque muitas pessoas com problemas de audição também apresentam dificuldades na fala. Pessoas com deficiência cognitiva têm dificuldade no reconhecimento e na retenção da informação, compreensão, envolvimento, identificação, escolha e implementação de soluções ou contextualização [KAVCIC, 2005]. As pessoas idosas geralmente encontram problemas de acesso quando o design de páginas apresenta uma estrutura que eles não conseguem entender, ou lhes incentivam distração com animações e dificuldade no manuseio do teclado e mouse [HANSON, 2004]. Além das barreiras citadas, existem outras como: idiomas, hardware e/ou software incompatível, ou problemas no ambiente onde o usuário está, como ambientes com muito barulho, com pouca ou muita luz.

\section{Regulamentações de acessibilidade Web}

A principal regulamentação sobre acessibilidade Web foi criada pelo W3C (World Wide Web Consortium), por meio das diretrizes constantes no documento WCAG (Web Content Accessibility Guidelines). Este documento é uma recomendação que estabelece pontos a serem considerados para que o conteúdo Web seja acessível. Ela está fundamentada em quatro princípios que um site deve ser: perceptível, operável, entendível e robusto. Na Tabela 1 é apresentado é um exemplo de recomendações de acessibilidade do WCAG 2.0 para o princípio perceptível. Essas recomendações são expandidas em critérios de sucesso que detalham o que deve ser feito para que a aplicação possa ser classificada como acessível. Cada critério de sucesso é descrito para determinar objetivamente se o conteúdo o satisfaz. Além disso, eles são classificados em três níveis: A, AA e AAA, de acordo com a importância do critério para a acessibilidade Web. O nível A é o nível mínimo e o nível AAA é quando se observa o máximo de acessibilidade. O WCAG 2.0 disponibiliza para cada critério de sucesso um conjunto de técnicas que explicam e exemplificam como implementar a acessibilidade para o mesmo [WCAG, 2008]. É importante notar também que pesquisas mais recentes estão divulgando a terminologia que deve ser adotada na redação de documentos científicos que tratem de acessibilidade [Cavender, 2008].

Tabela 1. Recomendações de acessibilidade do WCAG para um princípio.

\section{Perceptível}

1.1 Fornecer textos alternativos para conteúdo não-textual.

1.2 Fornecer alternativas para mídia baseada em tempo

1.3 Criar conteúdo que pode ser apresentado de diferentes formas.

1.4 Fazer o site mais fácil para os usuários verem e ouvirem o conteúdo incluindo separação do primeiro plano e plano de fundo. 
No Brasil a acessibilidade foi regulamentada por meio do Decreto-Lei 5.296 de 2 de dezembro de 2004 e as Leis $n^{\circ} 10.048$, de 8 de novembro de 2000 , que dá prioridade de atendimento às pessoas com necessidades especiais, e a $\mathrm{n}^{\circ} 10.098$, de 19 de dezembro de 2000, que estabelece normas gerais e critérios básicos para a promoção da acessibilidade. Este decreto, além de tratar da acessibilidade dos sites, faz indicações sobre outros meios de comunicação, transporte e arquitetura, para que todos os cidadãos brasileiros possam ter direitos iguais de acesso a tudo que for disposto a eles de maneira igualitária [BRASIL, 2004].

Outro esforço do governo brasileiro foi a criação, em janeiro de 2005, do e-MAG (Modelo de Acessibilidade do Governo Eletrônico) para auxiliar na padronização do processo de garantir acessibilidade dos sites e portais do governo eletrônico, e facilitar sua implementação [GOVERNO ELETRÔNICO, 2000]. Em maio de 2007, o e-MAG foi institucionalizado no âmbito do Sistema de Administração dos Recursos de Informação e Informática (SISP), tornando sua observância obrigatória nos sites e portais do governo brasileiro. O e-MAG se fundamenta nas regras do WCAG 1.0 e apresenta em documentos separados como a sua implementação pode ser percebida na visão do cidadão e na visão técnica.

A Visão do Cidadão separa os princípios de acessibilidade em áreas parecidas com os princípios do WCAG. São elas: percepção, operação, entendimento e compatibilidade. A Cartilha Técnica, que se refere à visão técnica, apresenta as diretrizes que reúnem as recomendações necessárias para serem implementadas nos sites acessíveis. As recomendações obedecem a níveis de prioridade. Os níveis de prioridade são utilizados para determinar o grau de acessibilidade da aplicação Web. Na Prioridade 1 estão as exigências básicas, sem elas, os usuários ficarão impossibilitados de acessar o conteúdo Web. Como Prioridade 2 constam normas e recomendações que garantem o acesso as informações do documento, caso contrário os usuários terão dificuldade no acesso e na Prioridade 3 estão normas e recomendações que, sendo implementadas, facilitarão o acesso aos documentos Web [e-MAG, 2005].

Apesar dos esforços para promover a acessibilidade, Ferreira et al. [2007] afirmam que a imposição do Decreto-Lei afetou muito pouco as organizações públicas brasileiras. Das 87 organizações que participaram da pesquisa em março de 2007, 22\% tinham seus sites adaptados, $31 \%$ estavam em adaptação e $47 \%$ dos sites não estavam adaptados. Destes sites não adaptados, 36,58\% das organizações conheciam o Decreto, $46,34 \%$ não conheciam, e as demais não souberam responder. Em outra pesquisa envolvendo 613 participantes ligados ao desenvolvimento de aplicações Web, distribuídos entre indústria, academia e governo, Freire et al. [2008] e Freire [2008], verificaram que 40,29\% dos participantes nunca ouviram falar do Decreto e 32,95\% já ouviram falar, mas não o conhecem ou conhecem vagamente.

Sendo assim, é necessária a conscientização sobre acessibilidade Web, seus benefícios e sua importância; e a divulgação da Lei de Acessibilidade Brasileira e do eMAG, pois somente a criação destes não é suficiente para promover a acessibilidade Web, como pode ser observado nas pesquisas acima. 


\section{Implementação da Acessibilidade Web nas organizações}

De acordo com o W3C [W3C, 2002], quando uma organização assume o compromisso de especificar e projetar um site acessível é importante que ela desenvolva um planejamento para a sua implementação. O W3C apresenta um conjunto de tarefas a serem cumpridas pela organização para implementação de acessibilidade, conforme apresentado na Tabela 2. O plano real de implementação, bem como a ordem das etapas a serem cumpridas, variam de organização para organização.

Tabela 2. Plano de implementação de acessibilidade nas organizações.

\section{Estabelecer responsabilidades}

1.1 Criar uma equipe de coordenação com um plano de comunicação.

1.2 Nomear um consultor de nível avançado ou um porta-voz para acessibilidade Web.

\section{Realizar uma avaliação inicial}

2.1 Informar sobre exigências externas com relação a acessibilidade Web as quais a organização deve cumprir ou está sujeita.

2.2 Conduzir avaliação inicial do site da organização usando o método da análise preliminar.

2.3 Avaliar a consciência atual da necessidade da acessibilidade dentro da organização, por meio de pesquisa ou entrevistas.

2.4 Avaliar experiência dos desenvolvedores Web da organização para projeto Web acessível.

2.5 Avaliar a capacidade dos softwares atuais no suporte ao desenvolvimento de sites acessíveis.

2.6 Estimar recursos requeridos para tratar as necessidades identificadas na avaliação inicial.

\section{Desenvolver a política da organização}

3.1 Identificar se a organização já possui uma política de desenvolvimento e de tecnologias para Web sites.

3.2 Estabelecer uma política de acessibilidade para a organização.

3.3 Desenvolver um plano para promover a conscientização para a política da organização, tanto internamente quanto externamente e que se aplique ao longo de todo o processo.

3.4 Divulgar a nova política da organização.

\section{Selecionar o Software}

4.1 Selecionar software de autoria que esteja de acordo com o ATAG.

4.2 Instalar configurações recomendadas que suportem a produção de conteúdo acessível.

4.3 Selecionar software para avaliação e para correção de acessibilidade.

4.4 Desenvolver um processo de publicação na Web que permita solucionar qualquer eventual inconsistência do software usado.

\section{Fornecer treinamento}

5.1 Planejar opções de treinamento para cobrir diferentes necessidades das pessoas com diferentes funções na organização.

5.2 Oferecer oportunidades constantes de treinamento que atendam às mudanças de equipe $\mathrm{e}$ de responsabilidades.

6 Desenvolver site acessível

6.1 Fazer da acessibilidade uma prioridade durante todo o processo de desenvolvimento.

6.2 Fornecer para meios à equipe de desenvolvimento de forma a assegurar acessibilidade.

7 Promover consciência de acessibilidade na organização

7.1 Incorporar a política de acessibilidade Web da organização nos documentos da organização, quando apropriado.

7.2 Regularmente reforçar a política de acessibilidade Web na organização.

8 Monitorar a acessibilidade Web

8.1 Especificar o processo de avaliação da acessibilidade Web e assegurar qualidade ao 
processo.

8.2 Monitorar o Web site da organização.

8.3 Fornecer feedback ao usuário sobre acessibilidade na organização.

8.4 Periodicamente rever todos os aspectos do plano de implementação para sua eficácia.

\section{Estudo de caso: melhoria de acessibilidade na GE (General Electric)}

Kumar [2007] aponta várias motivações para promover acessibilidade no site da General Electric, tais como: aproximadamente 20\% dos consumidores em potencial da GE são pessoas com limitações; princípios de design acessível melhoram a descoberta do conteúdo e independência dos serviços; aumento da diversidade de usuários; risco da não-conformidade com recomendações e leis de acessibilidade.

Antes de iniciar o processo de promover acessibilidade Web, o site da GE tinha um design simples, o que facilitou a melhoria da acessibilidade, porém, faltavam textos equivalentes para imagens e o site apresentava falta de estrutura, o que conduzia a uma tradução tediosa usando leitor de tela. Além disso, os formulários eram difíceis de completar e não era possível submetê-los sem clicar com o mouse no botão de submissão. Assim, a GE estabeleceu que seu site, ao final do processo de melhoria de acessibilidade, deveria estar em conformidade com as prioridades 1 e 2 do WCAG 1.0, Section 508 e Federação Nacional dos Cegos (NFB - National Federation of the Blind).

Uma vez verificados os pontos fracos que deveriam ser corrigidos e as metas a serem alcançadas, foram identificados os conteúdos vindos de templates, outros elementos comuns e o que deveria ser incluído no escopo. Após a definição do escopo, foi realizada uma avaliação automática do site GE existente e testes com tecnologias assistivas. O próximo passo foi priorizar o que tornava o acesso ao site impossível para pessoas com deficiência e identificar as partes do site que constituíam a funcionalidade essencial. Foram realizadas mudanças nos elementos comuns tais como templates, CSS e funções JavaScript. De acordo com Kumar [2007], incorporar acessibilidade aos templates foi essencial para assegurar a acessibilidade em todos os componentes do site. Durante a implementação da acessibilidade Web, verificou-se a dificuldade de minimização do site, e assim, foram encontrados desafios para tratar de assuntos como a remoção e adição de itens no site. Após a realização das mudanças, a versão acessível foi publicada. Novos conjuntos de desafios foram encontrados, como manter o nível de acessibilidade, que requer um constante processo de monitoria.

Ao final, a GE publicou um novo estilo de desenvolvimento e recomendações para serem aplicadas dentro da organização, por meio do estabelecimento de um processo de conformidade compreensível de acessibilidade Web.

\section{Conclusões}

O presente mini-curso tem como objetivo apresentar os principais fundamentos sobre acessibilidade e, principalmente, os passos e roteiro estabelecidos pelo W3C para institucionalização de acessibilidade em organizações.

A acessibilidade não deve ser considerada apenas no contexto de projetos isolados. É válido reforçar a importância de envolvimento de toda a equipe no projeto de fornecer soluções acessíveis. Sobretudo, o treinamento da equipe, a estimativa de 
recursos e o uso de ferramentas que estejam de acordo com as regulamentações devem ser valorizados.

Como principal benefício para organizações pode-se considerar o fato de que, ao entender a importância e as vantagens em termos de acessibilidade, a equipe terá condições de empregar os conceitos em outros setores da organização, por exemplo, na elaboração de documentos, e, como resultado, fornecer serviços que possam ser aproveitados de forma diferenciada.

Agradecimentos: Os autores agradecem ao CNPq (proc. 551017/2007-4) e Fundect (proc. 23/200.094/2008) pelo apoio financeiro.

\section{Referências}

BRASIL. Decreto-lei 5296, de 2 de dezembro de 2004. Regulamenta as Leis $\mathrm{n}^{\circ} \mathrm{s} 10.048$, de 8 de novembro de 2000 e 10.098, de 19 de dezembro de 2000. Publicado no D.O.U, no 232, 03 de dezembro de 2004.

CAVENDER, A., TREWIN, S.; H. VIRKI General writing guidelines for technology and people with disabilities, ACM SIGACCESS Accessibility and Computing, 2008, 17-22.

e-MAG. Recomendações de Acessibilidade para a Construção e Adaptação de Conteúdos do Governo Brasileiro na Internet. e-MAG, Acessibilidade de Governo Eletrônico. Modelo de Acessibilidade. Departamento de Governo Eletrônico, Secretaria de Logística e Tecnologia da Informação, Ministério do Planejamento, Orçamento e Gestão. Doc. de Ref., versão 2.0. 2005. 13 p.

FERREIRA, S. B.L., SANTOS, R.C., SILVEIRA, D., FERREIRA, M.G.L. Panorama da acessibilidade web brasileira. RCA - Revista de Controle e Administração. Publicação da Controladoria Geral do Município do Rio de Janeiro Vol. III,nº 2, jul./dez. 2007, p. 205 - 234.

FREIRE, A. P. Acessibilidade no Desenvolvimento de Aplicações Web: um Estudo sobre o Cenário Brasileiro. Janeiro de 2008. Dissertação de Mestrado. ICMC/USP. 134 p.

FREIRE, A. P.; FORTES, R. P. M.; RUSSO, C. M. The perception of accessibility in Web development by academy, industry and government: a survey of the Brazilian scenario. New Review of Hypermedia and Multimedia, v. 14, p. 149-175, 2008.

GOVERNO ELETRÔNICO. e-MAG - Governo Eletrônico. 2000. Disponível em: http://www.governoeletronico.gov.br/acoes-e-projetos/e-MAG. (Acesso em maio/2008)

HANSON, V. L. The User Experience: Designs and Adaptations. ACM International Conference Proceeding Series; Vol. 63. Proceedings of the 2004 international cross-disciplinary workshop on Web accessibility (W4A). New York City, New York, NY, USA. ACM, 2004. p. 1-11.

KAVCIC, A. Software Accessibility: Recommendations and Guidelines. Computer as a Tool, 2005. EUROCON 2005. The International Conference on. Vol. 2. Belgrade, Serbia and Montenegro. IEEE, 2005. p.1024 -1027.

KUMAR, P. Web Accessibility at General Electric. The Accessibility Imperative. Implications of the Convention on the Rights of Persons with Disabilities for Information and Communication Technologies. Edited by G3ict, Global Initiative for Inclusive Information and Communication Technologies. New York, Março 2007. p. 91-96.

PADDISON, C., ENGLEFIELD, P. Applying Heuristics to Perform a Rigorous Accessibility Inspection in a Commercial Context. ACM Conference on Universal Usability, Proceedings of the 2003 conference on Universal usability, Vancouver, British Columbia, Canada. ACM, 2003. p. 126-133.

PÜHRETMAIR, F., MIESENBERGER, K. Making sense of accessibility in IT Design - usable accessibility vs. accessible usability. 16th International Workshop on Database and Expert Systems Applications (DEXA'05), Copenhagen, Denmark. IEEE, 2005. p. 861-865.

W3C/WAI. Introduction to Web Accessibility. 1997. Disponível em: http://www.w3.org/WAI/intro/accessibility.php (Acesso em Janeiro/2008)

W3C. Implementation Plan for Web Accessibility. 2002. Disponível em: http://www.w3.org/WAI/impl/Overview.html (Acesso em Outubro/2002)

WCAG 2.0. Introduction to Understanding WCAG 2.0, 2008. Disponível em: http://www.w3.org/TR/2008/WD-UNDERSTANDING-WCAG20-20080430/conformance.html (Acesso em Setembro/2008) 